\title{
Parsing Arguments of Nominalizations in English and Chinese*
}

\author{
Sameer Pradhan, Honglin Sun, \\ Wayne Ward, James H. Martin \\ Center for Spoken Language Research, \\ University of Colorado, Boulder, CO 80303 \\ \{spradhan, sunh, whw, martin\}@cslr.colorado.edu
}

\author{
Dan Jurafsky \\ Department of Linguistics \\ Stanford University \\ Stanford, CA 94305 \\ jurafskyestanford.edu
}

\begin{abstract}
In this paper, we use a machine learning framework for semantic argument parsing, and apply it to the task of parsing arguments of eventive nominalizations in the FrameNet database. We create a baseline system using a subset of features introduced by Gildea and Jurafsky (2002), which are directly applicable to nominal predicates. We then investigate new features which are designed to capture the novelties in nominal argument structure and show a significant performance improvement using these new features. We also investigate the parsing performance of nominalizations in Chinese and compare the salience of the features for the two languages.
\end{abstract}

\section{Introduction}

The field of NLP had seen a resurgence of research in shallow semantic analysis. The bulk of this recent work views semantic analysis as a tagging, or labeling problem, and has applied various supervised machine learning techniques to it (Gildea and Jurafsky (2000, 2002); Gildea and Palmer (2002); Surdeanu et al. (2003); Hacioglu and Ward (2003); Thompson et al. (2003); Pradhan et al. (2003)). Note that, while all of these systems are limited to the analysis of verbal predicates, many underlying semantic relations are expressed via nouns, adjectives, and prepositions. This paper presents a preliminary investigation into the semantic parsing of eventive nominalizations (Grimshaw, 1990) in English and Chinese.

\section{Semantic Annotation and Corpora}

For our experiments, we use the FrameNet database (Baker et al., 1998) which contains frame-specific se-

This research was partially supported by the ARDA AQUAINT program via contract OCG4423B and by the NSF via grant IS-9978025 mantic annotation of a number of predicates in English. Predicates are grouped by the semantic frame that they instantiate, depending on the sense of their usage, and their arguments assume one of the frame elements or roles specific to that frame. The predicate can be a verb, noun, adjective, prepositional phrase, etc. FrameNet contains about 500 different frame types and about 700 distinct frame elements. The following example illustrates the general idea. Here, the predicate "complain" instantiates a "Statement" frame once as a nominal predicate and once as a verbal predicate.

Did [Speaker she] make an official [Predicate:nominal complaint] [Addressee to you] [Topic about the attack.]

[Message "Justice has not been done"] [Speaker he] [Predicate:verbal complained.]

Nominal predicates in FrameNet include ultra-nominals (Barker and Dowty, 1992), nominals and nominalizations. For the purposes of this study, a human analyst went through the nominal predicates in FrameNet and selected those that were identified as nominalizations in NOMLEX (Macleod et al., 1998). Out of those, the analyst then selected ones that were eventive nominalizations.

These data comprise 7,333 annotated sentences, with 11,284 roles. There are 105 frames with about 190 distinct frame role ${ }^{1}$ types. A stratified sampling over predicates was performed to select $80 \%$ of this data for training, $10 \%$ for development and another $10 \%$ for testing.

For the Chinese semantic parsing experiments, we selected 22 nominalizations from the Penn Chinese Treebank and tagged all the sentences containing these predicates with PropBank (Kingsbury and Palmer, 2002) style arguments - ARG0, ARG1, etc. These consisted of 630 sentences. These are then split into two parts: $503(80 \%)$ for training and $127(20 \%)$ for testing.

\footnotetext{
${ }^{1}$ We will use the terms role and arguments interchangeably
} 


\section{Baseline System}

The primary assumption in our system is that a semantic argument aligns with some syntactic constituent. The goal is to identify and label constituents in a syntactic tree that represent valid semantic arguments of a given predicate. Unlike PropBank, there are no hand-corrected parses available for the sentences in FrameNet, so we cannot quantify the possible mis-alignment of the nominal arguments with syntactic constituents. The arguments that do not align with any constituent are simply missed by the current system.

3.1 Features We created a baseline system using all and only those features introduced by Gildea and Jurafsky that are directly applicable to nominal predicates. Most of the features are extracted from the syntactic parse of a sentence. We used the Charniak parser (Chaniak, 2001) to parse the sentences in order to perform feature extraction. The features are listed below: Predicate - The predicate lemma is used as a feature. Path - The syntactic path through the parse tree from the parse constituent being classified to the predicate.

Constituent type - This is the syntactic category (NP, PP, $\mathrm{S}$, etc.) of the constituent corresponding to the semantic argument.

Position - This is a binary feature identifying whether the constituent is before or after the predicate.

Head word - The syntactic head of the constituent.

3.2 Classifier and Implementation We formulate the parsing problem as a multi-class classification problem and use a Support Vector Machine (SVM) classifier in the ONE vs ALL (OVA) formalism, which involves training $n$ classifiers for a $n$-class problem - including the NULL class. We use TinySVM ${ }^{2}$ along with $\mathrm{YamCha}^{3}$ (Kudo and Matsumoto $(2000,2001)$ ) as the SVM training and test software.

3.3 Performance We evaluate our system on three tasks: i) Argument Identification: Identifying parse constituents that represent arguments of a given predicate, ii) Argument Classification: Labeling the constituents that are known to represent arguments with the most likely roles, and iii) Argument Identification and Classification: Finding constituents that represent arguments of a predicate, and labeling them with the most likely roles. The baseline performance on the three tasks is shown in Table 1 .

\section{New Features}

To improve the baseline performance we investigated additional features that would provide useful information in identifying arguments of nominalizations. Following is a

\footnotetext{
${ }^{2}$ http://cl.aist-nara.ac.jp/ talus-Au/software/TinysVM/

${ }^{3}$ http://cl.aist-nara.ac.jp/ taku-Au/software/yamcha/
}

\begin{tabular}{||l|l|r|r|r|}
\hline \hline Task & $\begin{array}{l}\mathrm{P} \\
(\%)\end{array}$ & $\begin{array}{r}\mathrm{R} \\
(\%)\end{array}$ & $\mathrm{F}_{\beta=1}$ & $\begin{array}{r}\mathrm{A} \\
(\%)\end{array}$ \\
\hline \hline Id. & 81.7 & 65.7 & 72.8 & \\
Classification & - & - & - & 70.9 \\
Id. + Classification & 65.7 & 42.1 & 51.4 & \\
\hline \hline
\end{tabular}

Table 1: Baseline performance on all three tasks.

description of each feature along with an intuitive justification. Some of these features are not instantiated for a particular constituent. In those cases, the respective feature values are set to "UNK".

1. Frame - The frame instantiated by the particular sense of the predicate in a sentence. This is an oracle feature.

2. Selected words/POS in constituent - Nominal predicates tend to assign arguments, most commonly through postnominal of-complements, possessive prenominal modifiers, etc. We added the values of the first and last word in the constituent as two separate features. Another two features represent the part of speech of these words.

3. Ordinal constituent position - Arguments of nouns tend to be located closer to the predicate than those for verbs. This feature captures the ordinal position of a particular constituent to the left or right of the predicate on a left or right tree traversal, eg., first PP from the predicate, second NP from the predicate, etc. This feature along with the position will encode the before/after information for the constituent.

4. Constituent tree distance - Another way of quantifying the position of the constituent is to identify its index in the list of constituents that are encountered during linear traversal of the tree from the predicate to the constituent.

5. Intervening verb features - Support verbs play an important role in realizing the arguments of nominal predicates. We use three classes of intervening verbs: i) auxiliary verbs - ones with part of speech AUX, ii) light verbs - a small set of known light verbs: took, take, make, made, give, gave, went and go, and iii) other verbs - with part of speech VBx. We added three features for each: i) a binary feature indicating the presence of the verb in between the predicate and the constituent ii) the actual word as a feature, and iii) the path through the tree from the constituent to the verb, as the subject of intervening verbs sometimes tend to be arguments of nominalizations. The following example could explain the intuition behind this feature:

[Speaker Leapor] makes general [Predicate assertions] [Topic about marriage]

6. Predicate NP expansion rule - This is the noun equivalent of the verb sub-categorization feature used by Gildea and Jurafsky (2002). This is the expansion rule instantiated by the parser, for the lowermost NP in the tree, encompassing the predicate. This would tend to cluster NPs with a similar internal structure and would 
thus help finding argumentive modifiers.

7. Noun head of prepositional phrase constituents - Instead of using the standard head word rule for prepositional phrases, we use the head word of the first NP inside the PP as the head of the PP and replace the constituent type $\mathrm{PP}$ with $\mathrm{PP}-<$ preposition $>$.

8. Constituent sibling features - These are six features representing the constituent type, head word and part of speech of the head word of the left and right siblings of the constituent in consideration. These are used to capture arguments represented by the modifiers of nominalizations.

9. Partial-path from constituent to predicate - This is the path from the constituent to the lowest common parent of the constituent and the predicate. This is used to generalize the path statistics.

10. Is predicate plural - A binary feature indicating whether the predicate is singular or plural as they tend to have different argument selection properties.

11. Genitives in constituent - This is a binary feature which is true if there is a genitive word (one with the part of speech POS, PRP, PRP\$ or WP\$) in the constituent, as these tend to be markers for nominal arguments as in

[Speaker Burma 's] [Phenomenon oil] [Predicate search] hits virgin forests

12. Constituent parent features - Same as the sibling features, except that that these are extracted from the constituent's parent.

13. Verb dominating predicate - The head word of the first VP ancestor of the predicate.

14. Named Entities in Constituent - As in Surdeanu et al. (2003), this is represented as seven binary features extracted after tagging the sentence with BBN's IdentiFinder (Bikel et al., 1999) named entity tagger.

\section{Feature Analysis and Best System Performance}

5.1 English For the task of argument identification, features 2, 3, 4, 5 (the verb itself, path to light-verb and presence of a light verb), 6, 7, 9, 10 an 13 contributed positively to the performance. The Frame feature degrades performance significantly. This could be just an artifact of the data sparsity. We trained a new classifier using all the features that contributed positively to the performance and the $\mathrm{F}_{\beta=1}$ score increased from the baseline of $72.8 \%$ to $76.3 \%\left(\chi^{2} ; p<0.05\right)$.

For the task of argument classification, adding the Frame feature to the baseline features, provided the most significant improvement, increasing the classification accuracy from $70.9 \%$ to $79.0 \%\left(\chi^{2} ; p<0.05\right)$. All other features added one-by-one to the baseline did not bring any significant improvement to the baseline, which might again be owing to the comparatively small training and test data sizes. All the features together produced a classification accuracy of $80.9 \%$. Since the Frame feature is an oracle, we were interested in finding out what all the other features combined contributed. We ran an experiment with all features, except Frame, added to the baseline, and this produced an accuracy of $73.1 \%$, which however, is not a statistically significant improvement over the baseline of $70.9 \%$.

For the task of argument identification and classification, features 8 and 11 (right sibling head word part of speech) hurt performance. We trained a classifier using all the features that contributed positively to the performance and the resulting system had an improved $\mathrm{F}_{\beta=1}$ score of $56.5 \%$ compared to the baseline of $51.4 \%$ $\left(\chi^{2} ; p<0.05\right)$.

We found that a significant subset of features that contribute marginally to the classification performance, hurt the identification task. Therefore, we decided to perform a two-step process in which we use the set of features that gave optimum performance for the argument identification task and identify all likely argument nodes. Then, for those nodes, we use all the available features and classify them into one of the possible classes. This "two-pass" system performs slightly better than the "one-pass" mentioned earlier. Again, we performed the second pass of classification with and without the Frame feature.

Table 2 shows the improved performance numbers.

\begin{tabular}{||l|l|r|r|r|}
\hline \hline Task & $\begin{array}{l}\mathrm{P} \\
(\%)\end{array}$ & $\begin{array}{r}\mathrm{R} \\
(\%)\end{array}$ & $\mathrm{F}_{\beta=1}$ & $\begin{array}{r}\mathrm{A} \\
(\%)\end{array}$ \\
\hline \hline Id. & 83.8 & 70.0 & 76.3 & 73.1 \\
$\begin{array}{l}\text { Classification (w/o Frame) } \\
\text { Classification (with Frame) }\end{array}$ & - & - & - & - \\
$\begin{array}{l}\text { Id. + Classification } \\
\text { (one-pass, w/o Frame) }\end{array}$ & 69.4 & 47.6 & 56.5 & 80.9 \\
$\begin{array}{l}\text { Id. + Classification } \\
\text { (two-pass, w/o Frame) }\end{array}$ & 62.2 & 53.1 & 57.3 & \\
$\begin{array}{l}\text { Id. + Classification } \\
\text { (two-pass, with Frame) }\end{array}$ & 69.4 & 59.2 & 63.9 & \\
\hline \hline
\end{tabular}

Table 2: Best performance on all three tasks.

5.2 Chinese For the Chinese task, we use the one-pass algorithm as used for English. A baseline system was created using the same features as used for English (Section 3). We evaluate this system on just the combined task of argument identification and classification. The baseline performance is shown in Table 3.

To improve the system's performance over the baseline, we added all the features discussed in Section 4, except features Frame - as the data was labeled in a PropBank fashion, there are no frames involved as in FrameNet; Plurals and Genitives - as they are not realized the same way morphologically in Chinese, and Named Entities - owing to the unavailability of a Chinese Named Entity tagger. We found that of these features, 2, 3, 4, 6, 7 and 13 hurt the performance when added to the baseline, but the other features helped to some degree, although 
not significantly. The improved performance is shown in Table 3

\begin{tabular}{||l|l|r|r|}
\hline \hline Features & $\begin{array}{l}\mathrm{P} \\
(\%)\end{array}$ & $\begin{array}{r}\mathrm{R} \\
(\%)\end{array}$ & $\mathrm{F}_{\beta=1}$ \\
\hline \hline Baseline & 86.2 & 32.2 & 46.9 \\
Baseline & 83.9 & 44.1 & 57.8 \\
+ more features & & & \\
\hline \hline
\end{tabular}

Table 3: Parsing performance for Chinese on the combined task of identifying and classifying semantic arguments.

An interesting linguistic phenomenon was observed which explains part of the reason why recall for Chinese argument parsing is so low. In Chinese, arguments which are internal to the NP which encompasses the nominalized predicate, tend to be multi-word, and are not associated with any node in the parse tree. These violates our basic assumption of the arguments aligning with parse tree constituents, and are guaranteed to be missed. In the case of English however, these tend to be single word arguments which are represented by a leaf in the parse tree and stand a chance of getting classified correctly.

\section{Conclusion}

In this paper we investigated the task of identifying and classifying arguments of eventive nominalizations in FrameNet. The best system generates an $F_{1}$ score of $57.3 \%$ on the combined task of argument identification and classification using automatically extracted features on a test set of about 700 sentences using a classifier trained on about 6,000 sentences.

As noted earlier, the bulk of past research in this area has focused on verbal predicates. Two notable exceptions to this include the work of (Hull and Gomez, 1996) - a rule based system for identifying the semantic arguments of nominal predicates, and the work of (Lapata, 2002) on interpreting the relation between the head of a nominalized compound and its modifier noun. Unfortunately, meaningful comparisons to these efforts are difficult due to differing evaluation metrics.

We would like to thank Ralph Weischedel and Scott Miller of BBN Inc. for letting us use BBN's named entity tagger - IdentiFinder; Ashley Thornton for identifying the sentences from FrameNet with predicates that are eventive nominalizations.

\section{References}

[Baker et al.1998] Collin F. Baker, Charles J. Fillmore, and John B. Lowe. 1998. The Berkeley FrameNet project. In COLING/ACL-98, pages 86-90, Montreal.

[Barker and Dowty1992] Chris Barker and David Dowty. 1992. Non-verbal thematic proto-roles. In NELS-23, Amy Schafer, ed., GSLA, Amherst, pages 49-62.
[Bikel et al.1999] Daniel M. Bikel, Richard Schwartz, and Ralph M. Weischedel. 1999. An algorithm that learns what's in a name. Machine Learning, 34:211-231.

[Chaniak2001] Eugene Chaniak. 2001. Immediate-head parsing for language models. In $A C L$, Toulouse, France.

[Gildea and Jurafsky2000] Daniel Gildea and Daniel Jurafsky. 2000. Automatic labeling of semantic roles. In $A C L$, pages 512-520, Hong Kong, October.

[Gildea and Jurafsky2002] Daniel Gildea and Daniel Jurafsky. 2002. Automatic labeling of semantic roles. Computational Linguistics, 28(3):245-288.

[Gildea and Palmer2002] Daniel Gildea and Martha Palmer. 2002. The necessity of syntactic parsing for predicate argument recognition. In $A C L, \mathrm{PA}$.

[Grimshaw1990] Jane Grimshaw. 1990. Argument Structure. The MIT Press, US.

[Hacioglu and Ward2003] Kadri Hacioglu and Wayne Ward. 2003. Target word detection and semantic role chunking using support vector machines. In HLT, Edmonton, Canada.

[Hull and Gomez1996] Richard D. Hull and Fernando Gomez. 1996. Semantic interpretation of nominalizations. In $A A A I$ Conference, Oregon, pages 1062-1068.

[Kingsbury and Palmer2002] Paul Kingsbury and Martha Palmer. 2002. From Treebank to PropBank. In LREC-2002, Las Palmas, Canary Islands, Spain.

[Kudo and Matsumoto2000] Taku Kudo and Yuji Matsumoto. 2000. Use of support vector learning for chunk identification. In CoNLL-2000, pages 142-144.

[Kudo and Matsumoto2001] Taku Kudo and Yuji Matsumoto. 2001. Chunking with support vector machines. In NAACL.

[Lapata2002] Maria Lapata. 2002. The disambiguation of nominalizations. Computational Linguistics, 28(3):357-388.

[Macleod et al.1998] C. Macleod, R. Grishman, A. Meyers, L. Barrett, and R. Reeves. 1998. Nomlex: A lexicon of nominalizations.

[Pradhan et al.2003] Sameer Pradhan, Kadri Hacioglu, Wayne Ward, James Martin, and Dan Jurafsky. 2003. Semantic role parsing: Adding semantic structure to unstructured text. In ICDM, Melbourne, Florida.

[Surdeanu et al.2003] Mihai Surdeanu, Sanda Harabagiu, John Williams, and Paul Aarseth. 2003. Using predicateargument structures for information extraction. In $A C L$, Sapporo, Japan.

[Thompson et al.2003] Cynthia A. Thompson, Roger Levy, and Christopher D. Manning. 2003. A generative model for semantic role labeling. In ECML. 